\title{
Enhancing Critical Life-Cycle Decision Making in Complex Engineering Projects in the Context of Engineering Economy Courses
}

\section{Dr. K. Jo Min, Iowa State University}

K. Jo Min is Associate Professor and Associate Chair, Director of Undergraduate Education in Industrial and Manufacturing Systems Engineering Department at Iowa State University. He teaches courses on production systems, closed-loop supply chains, and engineering valuation. His education research interests include outcome assessment and visualization aids, and his engineering research focuses on application of stochastic optimal control on engineering decision making. He has co-authored numerous papers in The Engineering Economist, IEEE Transactions on Engineering Management, International Journal of Production Research, International Journal of Engineering Education, and other peer-reviewed journals. He has been serving as an ABET program evaluator for EAC and ETAC and as a reviewer for various NSF engineering education panels.

\section{Dr. John Jackman, Iowa State University}

John Jackman is an associate professor of industrial and manufacturing systems engineering at Iowa State University. His research interests include engineering problem solving, computer simulation, web-based immersive learning environments, and data acquisition and control.

Mr. Farshad Niayeshpour, Iowa State University 


\title{
Enhancing Critical Life-Cycle Decision Making in Complex Engineering Projects in the Context of Engineering Economy Courses
}

\begin{abstract}
Complex engineering projects (CEPs) such as electric transmission networks and transportation infrastructure are becoming increasingly important to the public in general and even more so to engineers. These projects are large-scale in terms of money and time and contain significant uncertainties over their life-cycle, with fluctuations in input and output costs. Due to these uncertainties, there are conditional opportunities (e.g., on prices) to make critical decisions such as investment in construction of power generation facilities or decommissioning of such facilities. Such decisions constitute strategic flexibilities or "real options" because the decision maker can alter the course of an investment over time when an uncertain aspect of the project such as the price becomes known. The current practice in engineering curricula, however, does not address the declarative and procedural knowledge necessary for critical economic decision making. We propose to (1) develop a module in an introductory course emphasizing the concept of the aforementioned strategic flexibilities and (2) develop an advanced course that is mathematically rigorous, yet with in-depth case studies for the CEPs. The module addresses the valuation of the strategic flexibilities over the life of CEPs to provide managerial insights and economic intuition. The advanced course emphasizes project experience including data-based parameter estimation and computation for optimal decisions. Both the module and course teaching materials will be complemented by a set of visualization aids for the key concepts and applications.
\end{abstract}

Keywords

Enhancing decision making, complex engineering projects, project valuation under uncertainty

\section{Introduction and Research Objective}

This project transforms the traditional teaching of engineering economics by using a stochastic optimal control perspective, where students will be introduced to the optimal threshold values for taking an action (e.g., the electricity price at which a generator may exit the market) as well as the optimal timing for such actions. These concepts of threshold and timing will have analytic forms without the pre-imposed granularity found in decision trees. Students will also learn to deal with confounding factors (e.g., Kirchhoff's law on electric transmission) and how best to synthesize them with the economics concepts, enhancing their insights and intuition. To our knowledge, there is little systematic and rigorous treatment of such flexibility in current engineering economy courses. Hence, this endeavor is expected to expand the current knowledge on the teaching and learning of the strategic flexibilities in CEPs in such courses.

This project aims to transform engineering economy education via a conceptual module in an introductory course and an experiential advanced course. Given that engineering economy courses are quite ubiquitous in colleges of engineering (taken by multiple engineering majors) across the U.S., if this project is successful, the potential impact of our findings on the teaching approach, teaching materials, and learning outcomes is truly substantial. Our methods of dissemination include journal papers and national conference presentations, and through these methods, we introduce our findings not only to traditional engineering economists, but also to teaching colleagues of project management in various disciplines, such as construction engineering. If this 
project is successful, then ultimately students of engineering economy will become better decision makers in CEPs that are becoming increasingly important in technology-driven societies, domestically and globally.

In what follows, we elaborate on the major goals of this project, followed by main activities and results, various impacts, and future directions.

The first goal of this project is to create engineering economy contents on CEP's suitable as relevant teaching materials under substantial uncertainties, emphasizing the threshold and timing of critical life-cycle decision making. The next goal is to facilitate many engineering students to be better able to make critical engineering economy decisions on CEP's through learning of relevant declarative knowledge through implementation of such materials as an elementary teaching module in an introductory engineering economy course. We also aim to facilitate select students to be better able to make critical engineering economy decisions on CEP's through learning of relevant declarative as well as procedural knowledge through implementation of such materials as a full course for advanced engineering economy under uncertainties. Finally, we aim to circulate the project results nationally and globally for a significant period of time via dissemination of such results in peer-reviewed, archival journals and conference proceedings as well as through conference presentations.

\section{Contents and Structure}

The main activities of this project can be summarized as follows: In Spring and Fall 2016 as well as in Spring and Fall 2017, the elementary teaching module was taught in IE 305, Engineering Economics Analysis (taught in an industrial engineering program). This course is required for all industrial engineering students and is used as a technical elective by students in other majors. In Fall 2015, Spring 2016, and Spring 2017, the experimental advanced full course, IE 405 Advanced Engineering Economy for Complex Engineering Projects, was taught. Engineering economy content on CEP's with substantial uncertainties to use as relevant and suitable teaching materials have been developed since August 2015 as such materials are simply rare thus far. As project results accumulated, the publication and dissemination efforts are becoming more extensive (relative to earlier months of this project).

All in all, we have made much progress towards the aforementioned goals. Specifically,

A. Creation, paper-writing, as well as publication efforts have focused on stochastic optimal control problems for electric power generation and transmission projects, engineering design projects, supply chain procurement projects.

B. The elementary teaching module was revised to become more compact and self-contained in IE 305 for Spring 2017 and Fall 2017, leading to a more effective and efficient introduction of decision making for CEP's to many students.

C. The advanced engineering economy course, IE 405, was taught in Spring 2017. This course is no longer experimental (that is, it was IE $405 \mathrm{X}$ in the previous year), and it is now more streamlined (peripheral discussion of math has been replaced by more relevant engineering applications in engineering design and other applicable areas). This has led to a more effective and efficient introduction of relevant declarative as well as procedural knowledge to select students. 
D. Significant dissemination efforts through papers and presentations have been made throughout the year.

Towards the aforementioned progresses $\mathrm{A}, \mathrm{B}, \mathrm{C}$, and $\mathrm{D}$, the following significant results are obtained.

A. Creation efforts contributed to four papers being published or in the pipeline [1] - [4]. One of which has obtained the 2nd Place winner of Manufacturing and Design Division Best Track Paper [3].

B. The teaching methodology and learning improvement are documented and shown in three published proceedings papers. [5] - [7].

C. The teaching methodology and learning improvement are being documented and to be summarized in a manuscript which is to be submitted to International Journal of Engineering Education soon.

D. In addition to $\mathrm{A}, \mathrm{B}$, and $\mathrm{C}$, we have participated/will participate in several national dissemination opportunities as follows: A multiple number of presentations have been made at the aforementioned ASEE, IISE, FIE, as well as INFORMS Conferences. Additionally, a poster presentation [8] was made by an EE major advised by K. J. Min at 2016 Innovative Smart Grid Technologies (ISGT) Conference, Minneapolis, MN.

Furthermore, we have successfully cast engineering design problems as complex engineering projects. The most substantial research findings are currently being documented in a manuscript, and it will be submitted to a relevant, major journal such as The Engineering Economist for a peer review in the very near future.

\section{Methodology}

In Spring 2017 and Fall 2017, the structure of the aforementioned elementary teaching module taught in IE 305 is as follows. The module consists of 4 class periods (50 minutes per period).

Period 1. A pre-test, traditional net present value approach, new questions under uncertainty such as flexible design.

Period 2. Using Min [9] (for Periods 2-4), introduction to GBM and Bellman optimality principle, hysteresis, optimal threshold to exit.

Period 3. Optimal expected remaining life. Sensitivity of the optimal solution, student contests.

Period 4. An epilogue with emerging application areas such as rare earth elements (REE's) under market uncertainties, further studies, and a post-test.

The elementary teaching module was taught in IE 305, Engineering Economics Analysis (taught in an industrial engineering program). This course is required for all industrial engineering students and is used as a technical elective by students in other majors. Our study used a single case design [10] recommended by the Department of Education, which does not require a control group because it focuses on the assessment of student understanding before and after an instructional intervention. The study was reviewed and approved by the Institutional Review Board (IRB). 
The three multiple choice questions covered the contents of the new teaching module. After students learned how to use the traditional Net Present Value approach to decision making, the pre-test was administered. The lectures previously described in the Module Contents and Structure section followed the pre-test. After the last lecture, the post-test was administered, which is the same as the pre-test. The tests were scored by assigning one point for each correct answer and no points for incorrect answers (i.e., a maximum score of 3).

We made each question to be self- explanatory within one page, and we added more instructions such as asking students to indicate their choices on the drawing itself, which was the main part of the question (rather than anywhere on the test paper).

Student subjects were mostly industrial engineering students in their junior or senior year with the. Other engineering disciplines included mechanical engineering, electrical engineering, chemical engineering, construction engineering, materials science and engineering, and computer engineering.

\section{Results and Analysis}

Two tailed paired t tests were performed for the differences in scores between the pre- and post-test using a significance level of 0.05 . The null hypothesis was that there was no effect on student learning and the alternative hypothesis is that there was an effect. Cohen's D statistic was used to quantify the effect size, if any, of the teaching module. Values of $0.2,0.5$, and 0.8 are considered to be small, medium, and large effects.

Table 1 paired t test results for each course offering

\begin{tabular}{|c|c|c|c|c|c|}
\hline \multirow{2}{*}{ Semester } & $\begin{array}{c}\text { Number of } \\
\text { Subjects }\end{array}$ & Questions & P-value & Cohen's D Value & Comparison to $\mathrm{H}_{\mathrm{o}}$ \\
\hline \multirow{2}{*}{ Spring 2017 } & \multirow{2}{*}{85} & $\mathrm{Q} 1$ & 0.009 & 0.336 & Reject $\mathrm{H}_{\mathrm{o}}$ \\
\cline { 3 - 6 } & & $\mathrm{Q} 2$ & $<0.001$ & 0.482 & Reject $\mathrm{H}_{\mathrm{o}}$ \\
\cline { 3 - 6 } & \multirow{2}{*}{ Fall 2017 } & $\mathrm{Q} 3$ & 0.580 & -0.077 & Cannot Reject $\mathrm{H}_{\mathrm{o}}$ \\
\cline { 3 - 6 } & 81 & $\mathrm{Q} 1$ & 0.096 & 0.388 & Cannot Reject $\mathrm{H}_{\mathrm{o}}$ \\
\cline { 3 - 6 } & & $\mathrm{Q} 2$ & 0.001 & 0.887 & Reject $\mathrm{H}_{\mathrm{o}}$ \\
\cline { 3 - 6 } & & $\mathrm{Q} 3$ & 0.537 & -0.151 & Cannot Reject $\mathrm{H}_{\mathrm{o}}$ \\
\hline
\end{tabular}

This is not surprising, given that question 2 usually had the poorest mean score for the pre-test as shown in Figure 1. Question 2 was used to assess student's understanding of the mathematical model and its impact on the timing of the decision.

In the following figure, we demonstrate the mean test scores for Spring 2017 and Fall 2017 course offerings. 


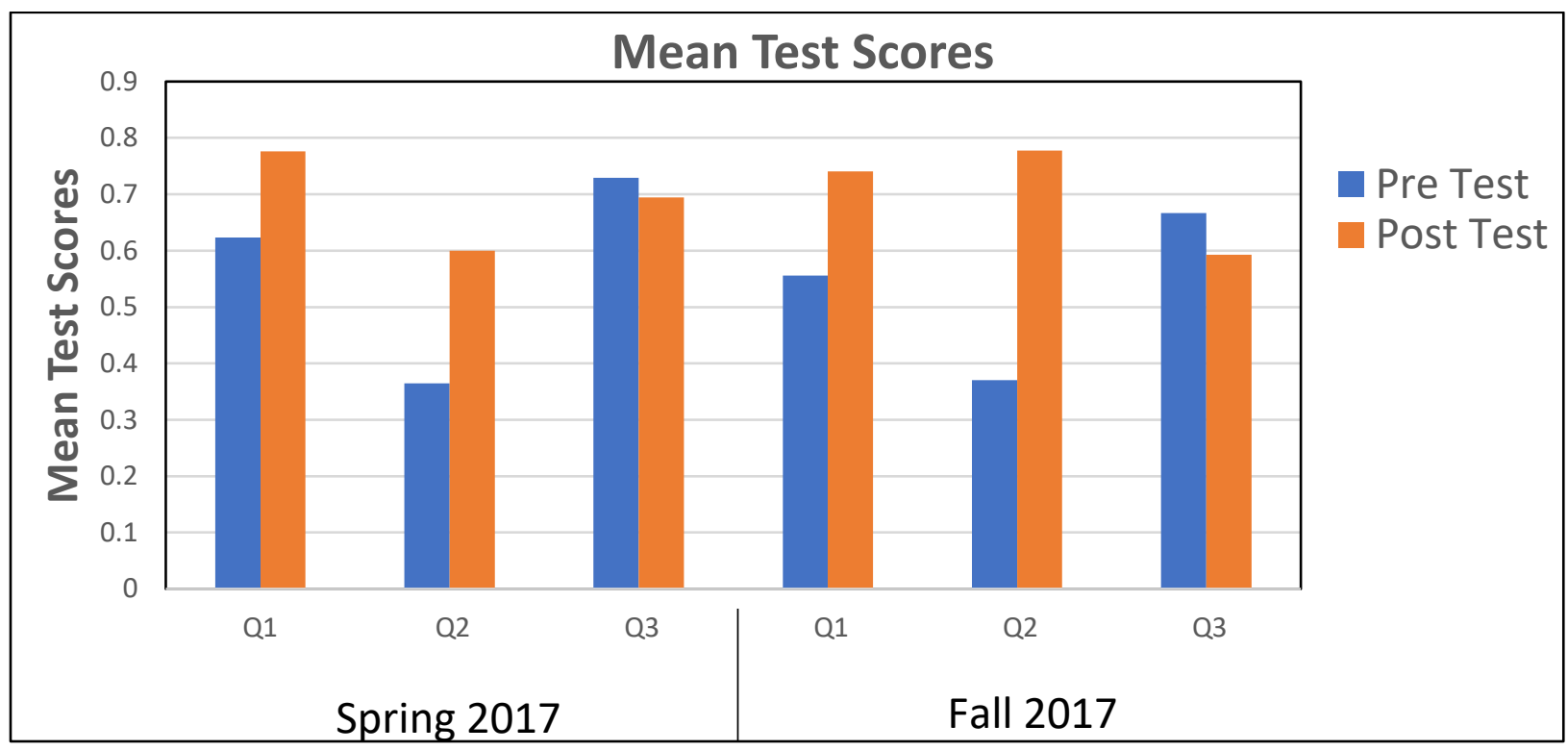

Figure 1 Mean scores for each question

\section{Concluding Remarks and Future Works}

As for impacts, first, we claim that engineering students at Iowa State University are now learning engineering economy with emphasis on the threshold and timing of decisions under substantial uncertainties based on stochastic optimal control. Moreover, such students are utilizing preliminary visual and tactile aids to learn industrial engineering topics funded by the supplementary REU of this project. Furthermore, we note that we currently have $1 \mathrm{PhD}$ and $8 \mathrm{BS}$ students (supplementary REU-funded) working in this project (which develops corresponding visual and tactile aids for better teaching and learning). They are acquiring knowledge, skills, and ability to collect and assess data, analyze statistically, and provide insights and guidelines.

Students who were taught and learned critical life-cycle decision making on complex engineering projects based on stochastic optimal control are equipped with a quantitative tool to address optimal decision making under uncertainties. For their professional careers as engineers, we think they are better prepared to address relevant uncertainties. For the graduate and undergraduate students who were direct participants in this project, they will have an innovative quantitative tool that addresses critical decision making under substantial uncertainties for their own future research and/or teaching.

Next, for the course contents on Complex Engineering Projects (CEP's), we note that both engineering design and supply chains can be modelled and analyzed as CEP's. Hence, a wide range of disciplines ranging from mechanical engineering to business and economics have new teaching materials and learning aids when complex decisions are to be made under substantial uncertainties.

Additionally, we think the applications to business, commerce, management, economics, and government are quite viable as explained in the second question above. With successful 
applications in such wide areas, we believe that the society will surely benefit from appropriate decision making in such complex problems under sizable uncertain.

Finally, in the near future, we will be crystallizing what we are going to cover by reducing peripheral math that students are unlikely to retain (e.g., non-central theorems and proofs) and by introducing more real-life examples found in engineering design problems as CEP's. We also plan to continue to produce relevant teaching materials and hope to find more generalizable principles (e.g., new ways of looking at engineering design as well as supply chain lead time challenges). Also, with the REU funding during Summer 2018, various visualization aids will be developed towards better engineering education research measured in terms of the accuracy of the knowledge attained and the lengthen of retention in memory. As before, all results will be documented and disseminated nationally and globally.

\section{References}

[1] Min, K. J. and F. Kucuksayacigil "Expansion Planning for Transmission Network under Demand Uncertainty: A Real Options Framework," The Engineering Economist. [Online]. Available: http://dx.doi.org/10.1080/0013791X.2016.1256459. Physical paper publication is expected in 2018.

[2] Min, K. J., J. Jackman, L. Lilienkamp, and C. Wang, "Supply Contracts for Critical and Strategic Materials of High Volatility and Their Ramifications for Supply Chains," The Engineering Economist. Under second review.

[3] F. Kucuksayacigil and Min, K. J., "Value of Jumboization in Ship Design: A Real Options Approach," in Proceedings of the 2017 Industrial and Systems Engineering Conference, Pittsburgh, PA, USA, 2017

[4] J. Croghan, Min, K. J., and J. Jackman, "Estimation of Geometric Brownian Motion Parameters for Oil Price Analysis," in Proceedings of the 2017 Industrial and Systems Engineering Conference, Pittsburgh, PA, USA, 2017.

[5] Min, K. J., J. Jackman, and M. Zugg, "Critical Life-Cycle Decision Making for Projects under Uncertainty," Engineering Economy Division, Proceedings of ASEE Annual Conference \& Exposition, New Orleans, Louisiana, USA, June 2016.

[6] Min, K. J., J. Jackman, and M. Zugg, "Economic Decision Making for Projects under High Volatility," Proceedings of the 2017 Industrial and Systems Engineering Conference, Pittsburgh, PA, USA, 2017

[7] Min, K. J., J. Jackman, and M. Zugg, "Visualization Aids for Abstract Concepts Towards Better Learning Outcomes," Proceedings of the 2017 Frontiers in Education (FIE), Indianapolis, IN, USA, 2017

[8] X. Guo, "Investment timing study for residential distributed energy resource projects: A real-option approach," in 2016 Innovative Smart Grid Technologies (ISGT) Conference, Minneapolis, MN, USA, 2016

[9] Min, K. J., C. Lou, and C. Wang, "An Exit and Entry Study of Renewable Power Producers: A Real Option Approach,” The Engineering Economist, Vol. 57, pp. 55-75. 2012

[10] T. Kratochwill, J. Hitchcock, R. Horner, J. Levin, S. Odom, D. Rindskopf, and W. Shadish, "Single-Case Designs Technical Documentation". What works clearinghouse, 2010 